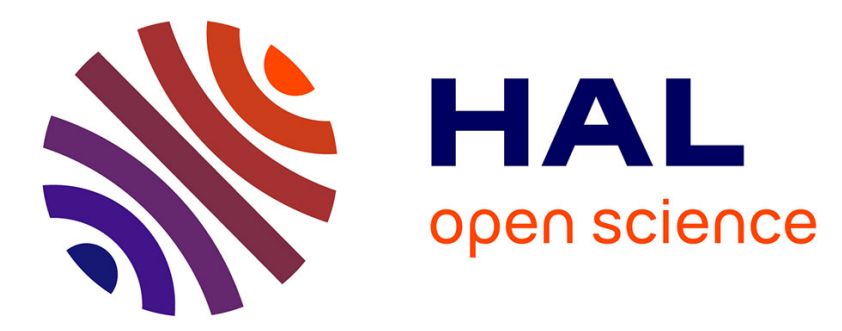

\title{
Une réfection des remparts de Philippes sous Michel VII Doukas
}

\author{
Samuel Provost
}

\section{To cite this version:}

Samuel Provost. Une réfection des remparts de Philippes sous Michel VII Doukas. Revue des études byzantines, 2003, 61, pp.167-182. 10.3406/rebyz.2003.2275 . hal-01292820

\section{HAL Id: hal-01292820 \\ https://hal.science/hal-01292820}

Submitted on 23 Mar 2016

HAL is a multi-disciplinary open access archive for the deposit and dissemination of scientific research documents, whether they are published or not. The documents may come from teaching and research institutions in France or abroad, or from public or private research centers.
L'archive ouverte pluridisciplinaire HAL, est destinée au dépôt et à la diffusion de documents scientifiques de niveau recherche, publiés ou non, émanant des établissements d'enseignement et de recherche français ou étrangers, des laboratoires publics ou privés.

\section{(이) $\$$}

Distributed under a Creative Commons Attribution - NonCommercial - NoDerivatives| 4.0 


\title{
UNE RÉFECTION DES REMPARTS DE PHILIPPES
}

\section{SOUS MICHEL VII DOUKAS*}

\author{
Samuel PROVOST
}

\begin{abstract}
Summary: An inscription recently discovered at Philippi constitutes the missing fragment of an inscription previously attributed to the walls of Drama. By combining the two texts we arrive at the date of 1077, at the end of the reign of Michael VII Doukas, for the restoration of the fortifications of the Western part of the city, on the initiative of the bishop Basil Kartzimopolos. This is the first precisely dated archeological confirmation of the existence of the site at the end of the eleventh century, which has also been established on the basis of literary sources.
\end{abstract}

Si l'existence d'un établissement byzantin sur le site de Philippes entre le $9^{\mathrm{e}}$ et le $14^{\mathrm{e}}$ siècle ne fait pas de doute, le débat reste encore ouvert sur son étendue et sa nature : le site a-t-il conservé sans discontinuer son caractère urbain, ou a-t-il été réduit à l'état d'une simple forteresse ou même d'un village fortifié ? Certaines sources littéraires mentionnent le site avec précision, mais la vérification archéologique de ces informations est longtemps demeurée problématique. Ainsi en est-il du passage du géographe arabe Idrîsî, qui dans son célèbre ouvrage, décrit Philippes au milieu du $12^{\mathrm{e}} \mathrm{s}$ comme une ville au commerce actif ${ }^{1}: \mathrm{M}$. Sève a pu écrire à bon droit encore très récemment que cette notice ne trouvait « aucun répondant dans l'archéologie $» 2$. Seuls peuvent en effet être datés de l'époque médiévale quelques chapelles aménagées dans les ruines des grandes basiliques paléochrétiennes et des travaux de fortification. Concernant les premières, E. Kourkoutidou-Nikolaïdou, qui en a dressé la liste ${ }^{3}$ pour montrer la continuité d'occupation du site à l'époque médiévale, ne peut guère les dater après le $11^{\mathrm{e}}$ siècle. La pauvreté architecturale de ces vestiges semble s'accorder à l'image de l'occupation du site réduite à un ou des établissements villageois plutôt qu'à une ville prospère.

\footnotetext{
* L'inscription dont il est question ici a été découverte dans le cadre des travaux menés à Philippes par l'École Française d'Athènes. Je remercie son directeur, D. Mulliez, d'avoir donné son accord pour cette publication dans la $R E B$. L'ensemble de la prospection du rempart byzantin donnera lieu par ailleurs à un article dans le $B C H$. Je remercie également D. Feissel et L. Foschia qui ont bien voulu me relire, et me faire part de leurs remarques sur une première version de cet article, dont $j$ 'assume naturellement l'entière responsabilité.

1. IDRîsî, La première géographie de l'Occident, éd. H. Bresc et A.-L. NeF, Paris 1999, p. 410.

2. M. SÈve, De la naissance à la mort d'une ville : Philippes en Macédoine (IVe siècle av. J.-C. $\mathrm{VII}^{\mathrm{e}}$ siècle ap. J.-C.), Histoire Urbaine no 1, juin 2000, p. 203 et n. 32. Le passage est plus longuement commenté par P. LEMERLe, Philippes et la Macédoine orientale, Paris 1945, p. 171 -174 (abrégé infra : LEMERLE, Philippes).

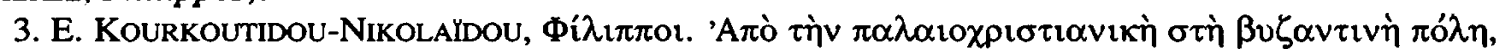

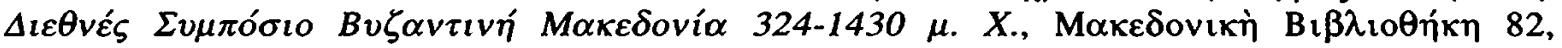
Thessalonique 1995, p. 171-182.
}

Revue des Études Byzantines 61, 2003, p. 167-182 


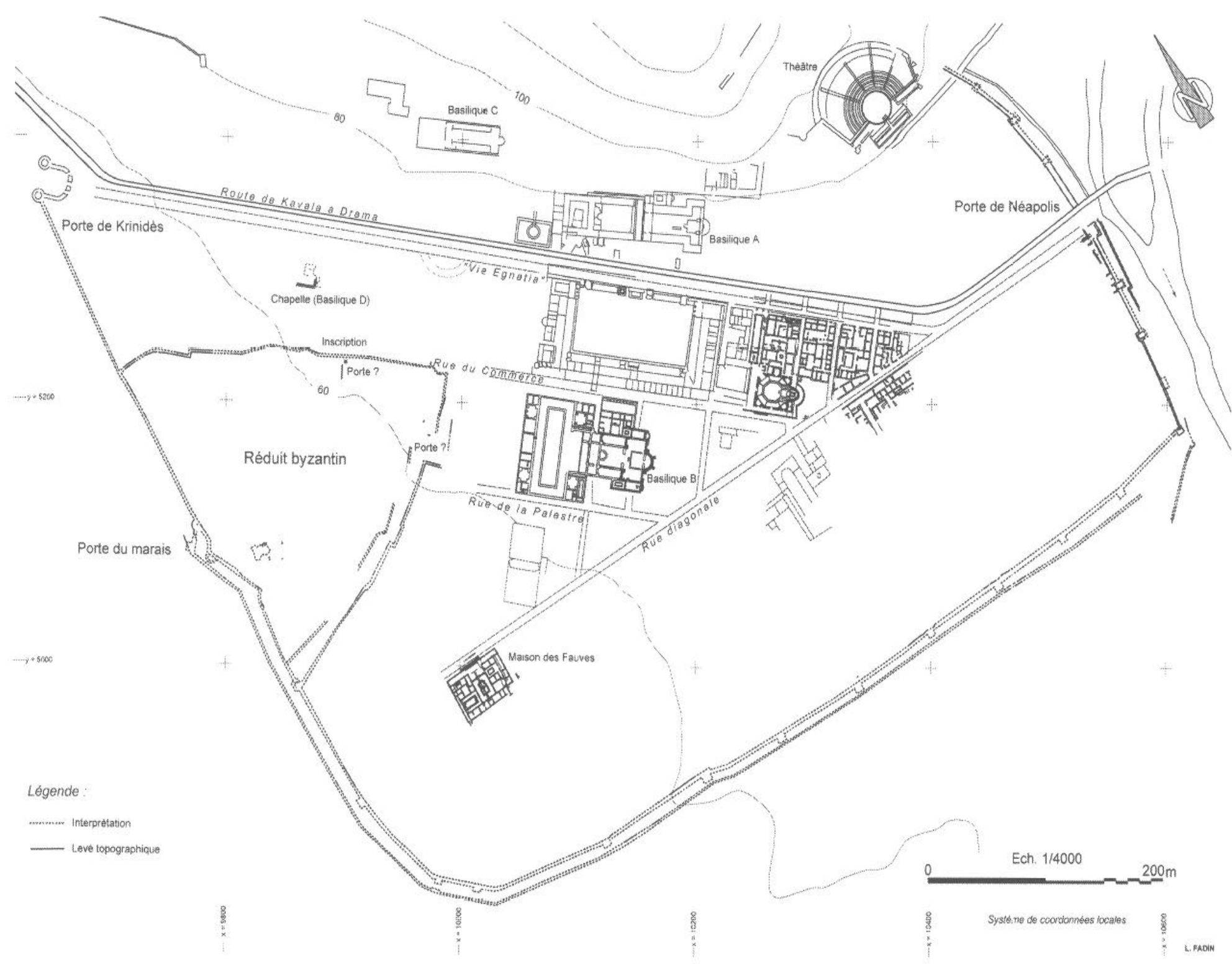

Fig. 1 - Philippes, plan de la ville basse et du réduit byzantin 
Les fortifications byzantines de Philippes ne permettent pas davantage d'éclaircir ce problème, tant la chronologie en est également incertaine et débattue. La seule inscription byzantine connue jusqu'à présent sur le site s'y rapporte pourtant bien : trouvée par P. Lemerle 4 en 1937, en remploi dans une maison du village contemporain voisin de Raktscha/Krinidès, elle commémore une réfection du kastron sous le règne de Nicéphore Phocas, entre 963 et 969 . Il est généralement admis qu'elle provient des fortifications de l'acropole, plutôt que de l'enceinte basse ${ }^{5}$. Mais l'hypothèse de $P$. Lemerle datant de cette époque une reconstruction complète des fortifications urbaines ne tient plus ${ }^{6}$. Des recherches récentes ${ }^{7}$ vont jusqu'à remettre en cause l'existence d'une réfection des remparts à l'époque mésobyzantine en dehors de l'acropole, dont le « château » est par ailleurs beaucoup plus récent. L'inscription du $10^{\mathrm{e}} \mathrm{s}$. ne pourrait ainsi être attribuée à aucun des importants vestiges encore visibles, qui appartiendraient soit à l'enceinte de l'Antiquité tardive, soit à ses dernières réfections byzantines.

Ce tableau est toutefois quelque peu modifié par une nouvelle découverte épigraphique. On a récemment identifié autour d'une porte de la courtine Ouest de l'enceinte, la « Porte du Marais » (Fig. 1), un système de défenses secondaires délimitant un réduit fortifié 8 daté, notamment d'après la céramique présente en surface, entre le $10^{\mathrm{e}}$ et le $14^{\mathrm{e}} \mathrm{s}$. Dans une haie masquant le remblai du rempart nord de ce réduit, a été découvert un bloc portant une inscription byzantine inédite (Fig. 2). Bien que la pierre n'ait pas été retrouvée à proprement parler in situ, et qu'elle ait donc pu être déplacée, sa localisation sur le talus résultant de l'effondrement du rempart reste le meilleur indice sur son emplacement originel, indépendamment du contenu de l'inscription.

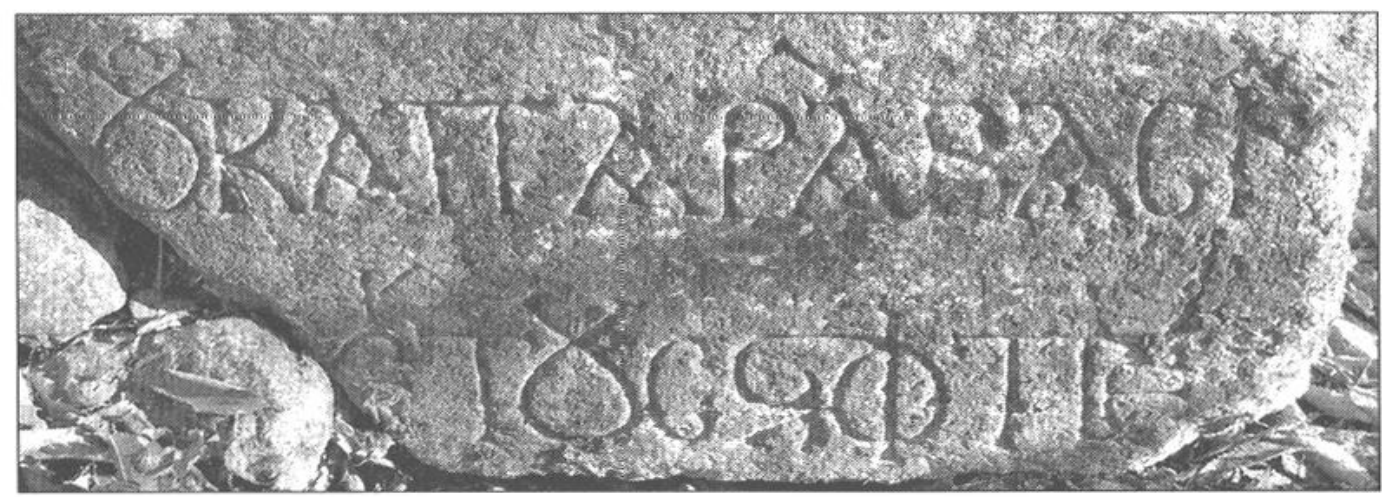

Fig. 2 - inscription de Philippes

4. P. Lemerle, Le château de Philippes au temps de Nicéphore Phocas, $B C H$ 61, 1937, p. $103-108$.

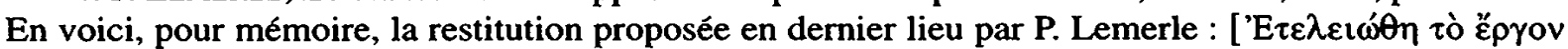

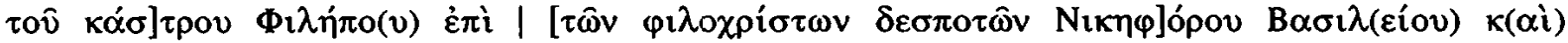

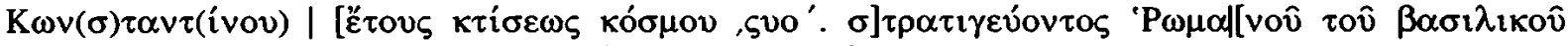

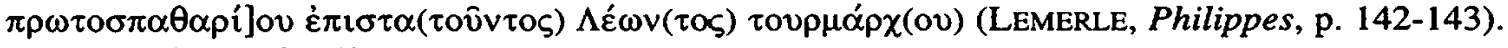

5. Ibid. p. 148-150.

6. Voir M. SĖVE, op. cit. p. 192.

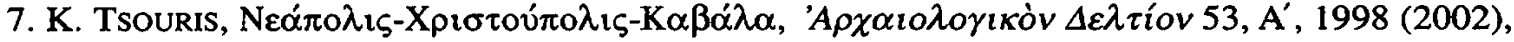
p. 433 : l'a. cite à l'appui de cette thèse une étude inédite de N. Zikos.

8. En attendant la publication à venir de la prospection topographique du réduit, voir les premiers résultats publiés dans : S. Provost, M. BOYD, Application de la prospection géophysique à la topographie urbaine, II. Philippes, les quartiers Ouest, $B C H$ 126/2, 2002, p. $435-447$ particulièrement. 
Il s'agit d'un parpaing de marbre local, brisé sur le côté gauche, travaillé sur une des faces de parement et de joint, ainsi que sur la face inférieure : celle-ci comprend un angle droit évidé muni d'un trou circulaire d'environ $18 \mathrm{~cm}$ de diamètre, plusieurs fois recreusé (Fig. 3). Ce dispositif correspond sans aucun doute à la feuillure intérieure d'un linteau de porte, avec dans l'angle la crapaudine destinée à recevoir le tourillon du pivot d'un vantail. Deux mortaises rectangulaires sont en outre visibles sur la face supérieure. Il est certes possible que l'inscription ait été gravée sur un bloc en remploi. Mais la taille, la nature du bloc, comme son emplacement, laissent peu de doute sur son identification à l'extrémité droite d'un linteau de porte monumentale.

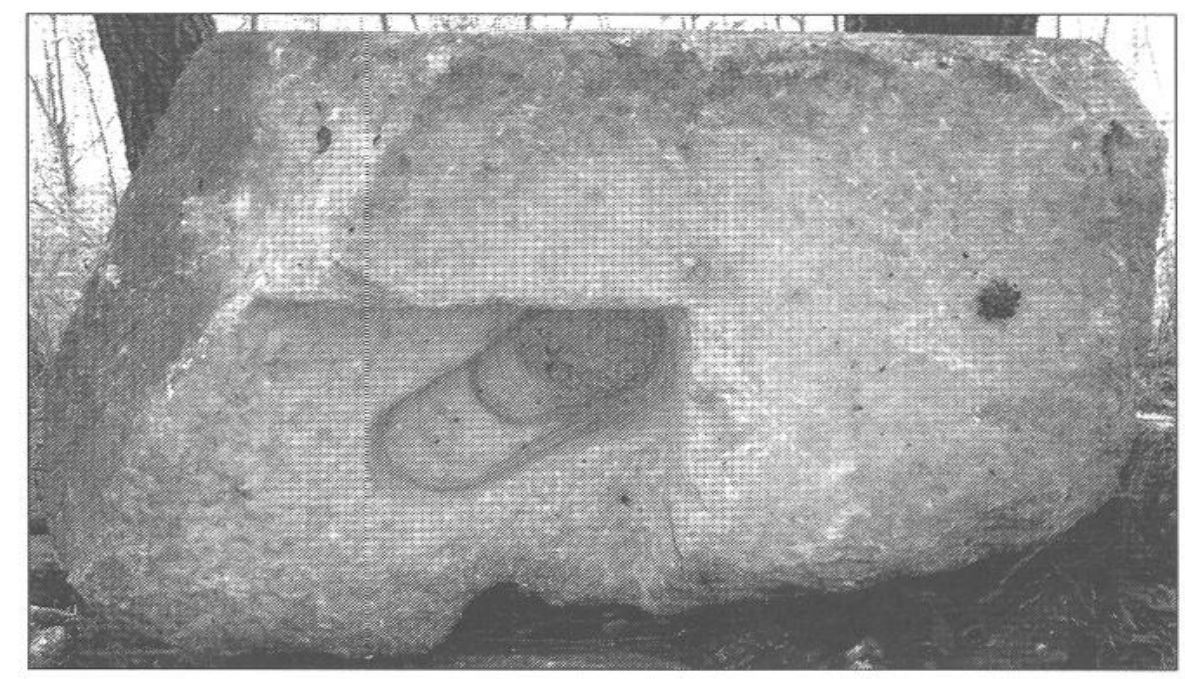

Fig. 3 - face inférieure du bloc portant l'inscription de Philippes

Bloc de marbre, brisé sur son côté gauche, et légèrement endommagé sur son côté droit. Hauteur : 0, $35 \mathrm{~m}$. Longueur : 0,80 m. Largeur : 0,50 m.

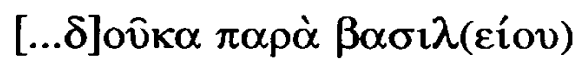

$$
\begin{aligned}
& \text { [ ] हैं }
\end{aligned}
$$

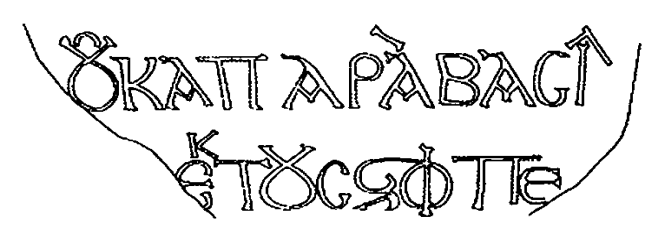

Lettres d'environ $7 \mathrm{~cm}$ de haut. Majuscules soigneusement gravées et accentuées. Ligatures $\succ$ lignes 1 et 2 . Lettres superposées $ı \wedge$ ligne 1 marquant une abréviation.

Les lettres se caractérisent par des formes souvent arrondies, notamment pour les $K, P$, $C, \epsilon$ et le $A$ surmonté d'un apex tourné vers la gauche. Cette particularité du A, qui se retrouve sur d'autres lettres, notamment dans l'inscription de Drama, peut constituer un indice chronologique 9 pour une datation aux $10^{\mathrm{e}}-11^{\mathrm{e}}$ siècle Les lettres droites, $1, \Pi$ et $\mathrm{T}$ présentent un léger empattement aux extrémités des hastes.

9. Voir l'exemple très proche par sa date et son objet d'une inscription de la porte d'Apros, en Thrace, sous Constantin X (1064), et les parallèles donnés par l'éditeur : C. AsDRACHA, Inscriptions de

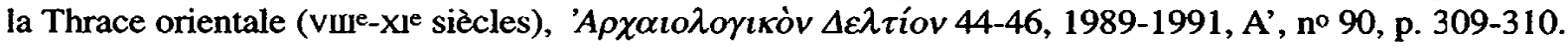


L. 1. L'accentuation est cruciale pour tenter de comprendre les deux mots fragmentaires. Pour le premier, si la restitution d'un delta initial ne pose pas de problème, il reste encore à trancher entre le nom propre et le titre auxquels ce $\delta$ ov̂k $\alpha$ peut renvoyer.

Dans la littérature comme dans les sources épigraphiques, l'accentuation habituelle du nom de famille des Douka impose un accent aigu au génitif $(\Delta o v ́ \kappa \alpha)^{10}$ qui serait attendu ici. Néanmoins, probablement parce que le nom tire son origine du titre ${ }^{11}$ de $\delta o v ́ \xi$, les sources emploient parfois $\Delta$ ov́ $\xi$ en lieu et place du

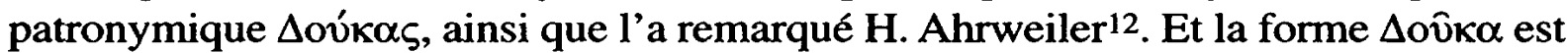
ainsi attestée chez certains auteurs comme Cedrenus ${ }^{13}$. On ne peut donc exclure qu'il s'agisse bien ici de ce patronyme. Mais la forme correspond de loin le plus fréquemment à l'accusatif du titre de $\delta$ ov́ $\xi$, raisons pour laquelle nous restituons dans un premier temps le $\delta$ minuscule.

La dernière lettre de la première ligne pose un problème de lecture en raison des accidents de la pierre. Il peut s'agir soit d'un $I$, soit d'un $H$ auquel manquerait la deuxième barre verticale, si le trait oblique final n'est pas accidentel ${ }^{14}$. Il est aussi possible d'y lire une marque d'abréviation, solution ici retenue. Au-dessus du I, se lit un petit $\wedge$. Le problème de savoir si le mot ainsi abrégé correspond à

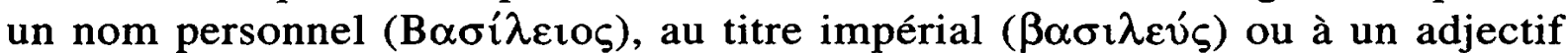
( $\beta \alpha \sigma \imath \lambda$ ıкós ou $\left.\beta \alpha \sigma i ́ \lambda \varepsilon \imath \_\varsigma\right)$ ne peut être résolu de façon satisfaisante dans l'état fragmentaire de l'inscription. L'absence d'article défini et le caractère peu probable d'une mention de l'empereur en toute fin d'un texte de ce type font préférer la première hypothèse.

L. 2. Le $\in$ final ne fait pas de doute, bien qu'il soit quelque peu tassé contre le $\Pi$ précédent, probablement faute de place sur la pierre, l'angle inférieur droit étant cassé.

La date se lit donc 6585 en ère mondiale byzantine (=1076/1077 ap. J.-C. $)^{15}$.

Ce fragment épigraphique peut être rapproché sans difficulté des formulaires d'inscriptions monumentales les plus courants à l'époque mésobyzantine. Il s'agit en effet selon toute probabilité d'une inscription commémorant l'achèvement de travaux de construction à Philippes. Le formulaire pourrait être ainsi analogue à celui de l'inscription du katépan de Thessalonique Constantin Diogène en Piérie16,

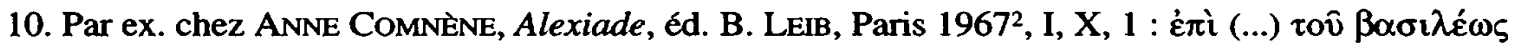

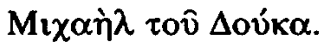

11. D. Polemis, The Doukai, Londres 1968, p. 3. Bryennios, historien du règne de Michel VII, rapporte lui-même une tradition en ce sens, faisant remonter l'origine de la famille à l'époque de Constantin le Grand (éd. Bonn, p. 13).

12. H. GlYKATZI-AHRWEILER, Recherches sur l'administration de l'empire byzantin aux LXe-XIe siècles, $B C H 84,1960$, p. 55 , n. 1. L'a. y mentionne notamment le cas de Constantin Doukas, cousin de l'empereur Michel VII, parfois appelé $\Delta$ oú $\xi$ par Bryennios ou Psellos.

13. Par ex. pour Constantin Doukas, Georgius Cedrenus, Chronogr. Compendium historiarum, éd. Bonn, vol. 2, page 2887 .

14. Le rapprochement avec l'inscription de Drama (voir infra), de graphie très proche, semble exclure toutefois cette hypothèse puisque la barre transversale des $\mathrm{H}$ y est droite.

15. V. GRUMEL, La chronologie, Paris 1958, p. 255.

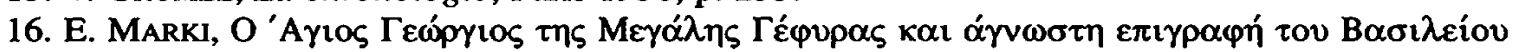

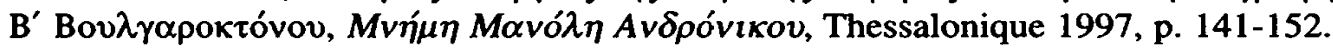


datée de 1015, pour prendre un exemple chronologiquement et géographiquement

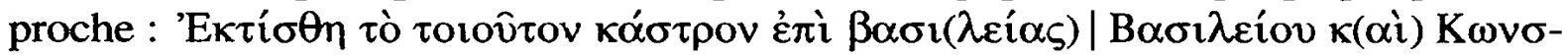

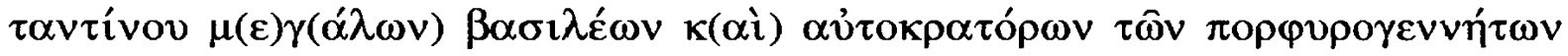

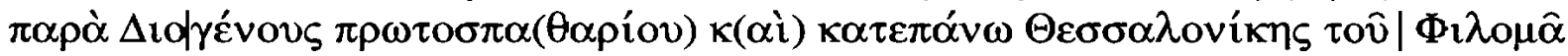

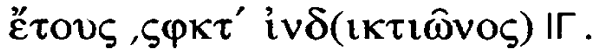

La partie manquante du texte de Philippes qui figurait sur le prolongement des deux lignes vers la gauche devait ainsi indiquer, sur la première ligne, la nature des travaux (construction ou réfection, complète ou limitée à un élément précis, tour, porte, etc.), l'empereur régnant, et sur la deuxième ligne l'identité et les titres du ou des responsables locaux. Il faut donc supposer que la partie manquante de l'inscription était d'une longueur au moins double de celle du fragment conservé.

Or une inscription (Fig. 4) découverte lors de travaux de voirie à Drama en 1985 correspond de façon frappante aux renseignements qu'on attendrait de la partie manquante du texte de Philippes (Fig. 5). Actuellement exposée au musée de Drama, elle a fait l'objet de deux publications 17 indépendantes l'une de l'autre, qui en donnent presque la même lecture, mais proposent des conclusions radicalement différentes sur sa datation.

Bloc de marbre brisé. Hauteur : 0,35 m. Longueur : 2,38 m. Épaisseur : 0, $57 \mathrm{~m}$. Hauteur des lettres : 7-8 cm.
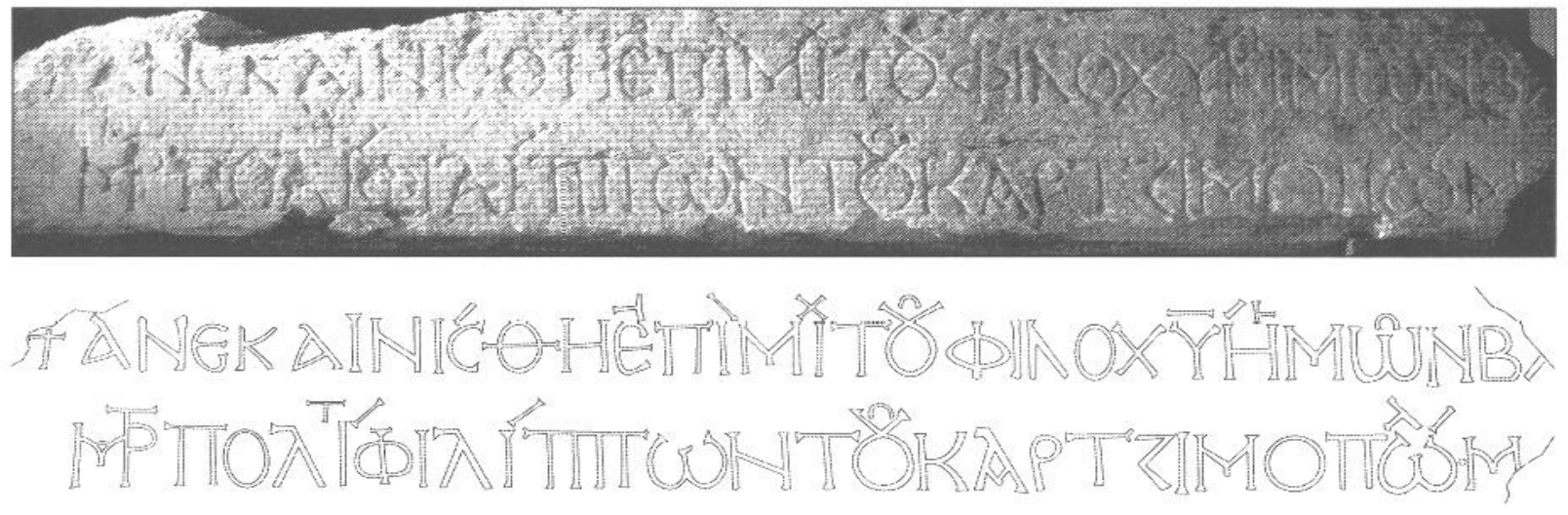

Fig. 4 - Inscription de Drama
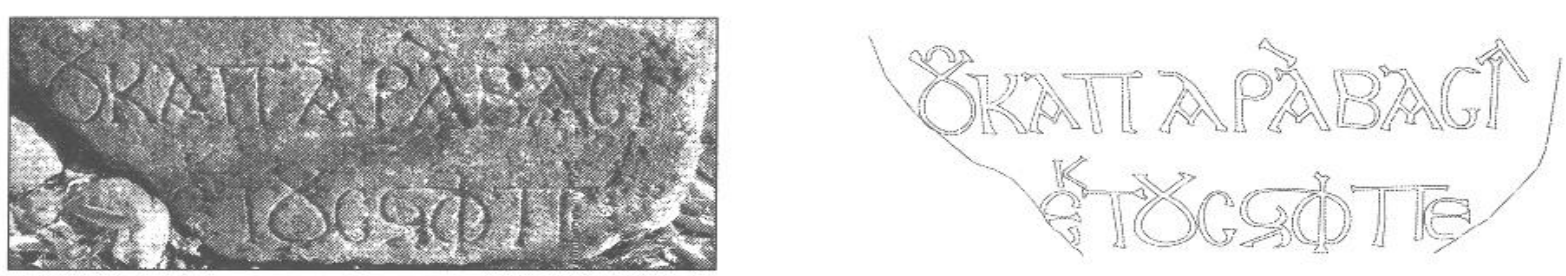

Fig. 5 - fragment de Philippes

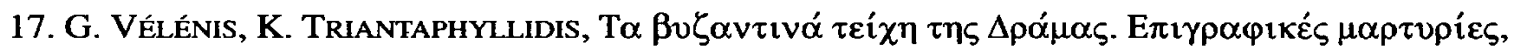
Byzantiaka 11, 1991, p. 99-116 (abrégé par la suite VélÉNIS-TRIANTAPHYLLIDIS, Drama). L'inscription a été concurremment publiée par N. ZIKos, assortie d'un bref commentaire, dans 'A $\Delta \varepsilon \lambda \tau i ́ o v ~ 40,1985$, Chron. p. 281-282. Les deux éditions sont assorties d'un fac-similé de l'inscription, mais seule celle de Vélénis et Triantaphyllidis en donne une photographie, d'assez médiocre qualité. 


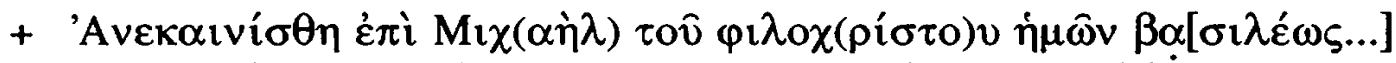

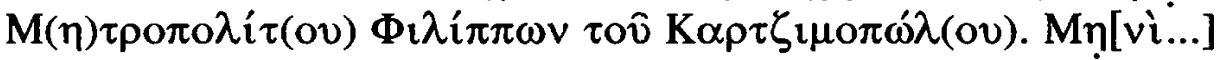

Accents et esprits indiqués. Ponctuation marquée avant la date. Ligatures M(H)TP, $\succ$ (deux fois), $\Pi T$. Lettres superposées marquant une abréviation : $I X, I T, \omega \wedge$. Abréviation $\chi(\rho i ́ \sigma \tau)$ v avec une barre au-dessus du upsilon et l'accent décalé à droite. Deux formes utilisées pour les lettres $A$ et $\wedge$, une petite variation dans celle du $\omega$.

L. 1. N. Zikos a omis une partie de l'accentuation (les deux esprits) sur le fac-similé qu'il donne de l'inscription.

L. 2. G. Vélénis et $K$. Triantaphyllidis n'ont pas lu le petit tau superposé de $M(\eta) \tau \rho o \pi o-$

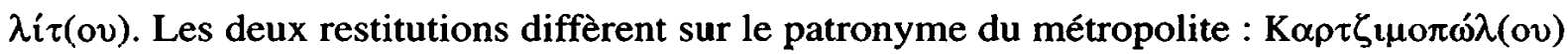

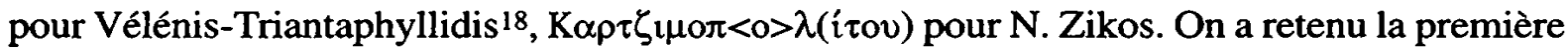
solution qui n'impose pas de correction orthographique.

Les éditeurs de cette inscription ont essayé en vain de retrouver le fragment droit manquant de la pierre, dont ils supposent qu'il devait porter le nom de l'évêque, à la première ligne, et la date de l'achèvement des travaux, à la seconde'19. Ces éléments sont précisément ceux que donne l'inscription de Philippes, en y ajoutant un possible patronyme impérial.

Un des arguments décisifs pour le rapprochement des deux pierres tient dans la comparaison des écritures, très proches, malgré quelques variations de détail : les quatre occurrences du alpha sur la pierre de Philippes n'utilisent qu'une des deux formes repérées pour cette lettre sur celle de Drama ; le sigma est davantage fermé sur le fragment de Philippes. Mais ces différences sont mineures, puisque pour le reste, les écritures présentent des caractéristiques identiques, jusque dans le détail de l'accentuation ou des abréviations notamment.

S'il était encore permis de douter de la complémentarité de ces deux fragments, l'examen du bloc retrouvé à Drama lève toute ambiguîté : sa face inférieure présente en effet un travail identique à celui observé sur le bloc de Philippes, à savoir une feuillure intérieure et une crapaudine. Les deux éditeurs de l'inscription y ont donc reconnu sans hésiter la partie gauche d'un linteau de porte.

Les dimensions des deux pierres, leurs caractéristiques, comme celles des inscriptions, permettent de conclure qu'il s'agit des deux parties du même linteau. Il portait donc un texte que l'on peut restituer de la manière suivante :

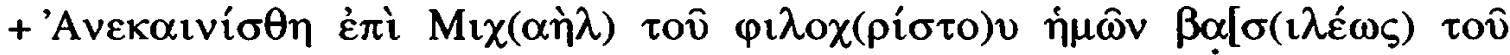

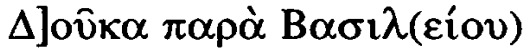

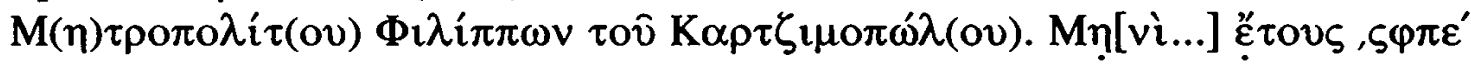

A été restauré sous Michel Doukas notre empereur aimant le Christ par Basile Kartzimopôlos, métropolite de Philippes, au mois de ... l'an 6585 (=1076/1077).

18. Leur transcription donne en fait $K \alpha \rho \tau \iota \zeta \mu o \pi \omega ́ \lambda(o v)$ mais le premier iota est une erreur typographique, comme le commentaire le montre par la suite : VÉlÉNIS-TRIANTAPHYLLIDIS, Drama, p. 100-101.

19. Ibid. 
Les lacunes entre les deux textes causées par la cassure du lintėau ne demandent qu'une restitution minimale. Ligne 1 , il suffit en effet de 4 lettres supplémentaires, si l'on suppose que le titre impérial était abrégé normalement en $\beta \alpha \sigma\left(\imath \lambda \varepsilon c^{\omega} \omega \varsigma\right)$, et que l'article présentait la ligature $\succ$ habituelle. Ligne 2, la lacune est un peu plus importante en raison de la cassure oblique de la pierre de Philippes, qui se prolonge sous les deux premières lettres de la ligne $1(\measuredangle K)$ : on peut donc restituer 6 lettres supplémentaires, soit les deux lettres finales de $\mu \eta v i$ et l'abréviation en quatre lettres d'un nom de mois, ou encore si la date était plus développée, la mention de l'indiction $15^{20}$, $\mu \eta v i ́$ étant alors abrégé.

Chaque ligne compterait ainsi un nombre équivalent de caractères.

Faute de disposer de la fin du texte, les éditeurs de l'inscription de Drama arrivaient à deux datations très différentes. Selon N. Zikos ${ }^{21}$, elle s'inscrivait dans le mouvement de refortification des villes de Macédoine aux $9^{\mathrm{e}}-10^{\mathrm{e}} \mathrm{s}$. attesté par l'épigraphie pour Thessalonique, Philippes et Kavala ${ }^{22}$ : il la datait ainsi du $9^{\mathrm{e}}$ s., faute de l'existence d'un empereur nommé Michel au siècle suivant, sans avoir envisagé l'hypothèse d'une datation plus tardive. G. Vélénis et $\mathrm{K}$. Triantaphyllidis s'appuyaient pour leur part sur l'analyse de l'onomastique, les particularités de cette écriture monumentale très soignée, et enfin, naturellement sur la mention d'un empereur Michel. Les premiers éléments leur faisaient préférer une date plus tardive, au $11^{\mathrm{e}} \mathrm{s}$. Ils parvenaient ainsi à proposer une date ${ }^{23}$ comprise entre 1034 et 1078 , sous le règne d'un des quatre empereurs prénommés Michel au $11^{\mathrm{e}} \mathrm{s}$. Le fragment de Philippes confirme pleinement leur analyse et précise que l'inscription fut gravée sous l'avant-dernière année du règne de Michel Doukas.

L'objet des travaux n'est pas nommé, mais une telle inscription monumentale ne peut guère correspondre à l'époque mésobyzantine qu'à deux types d'édifices, églises ou fortifications. Or ainsi que le soulignent G. Vélénis et K. Triantaphyllidis, le linteau monolithique qui porte l'inscription impressionne par sa taille 24 : ils calculent très justement qu'elle devait atteindre entre 3,30 et $3,50 \mathrm{~m}$, pour une embrasure de porte probablement comprise entre $2,30 \mathrm{~m}$ et $2,50 \mathrm{~m}^{25}$. Une telle ouverture est incompatible avec la taille habituelle des églises mésobyzantines. D'autre part, le diamètre important de la crapaudine creusée dans le linteau implique que le vantail pouvait avoir une épaisseur de 0,16 à $0,18 \mathrm{~m}$, ce qui con-

20. Voir l'inscription d'Apros mentionnée (supra, n. 9) pour une date comparable, avec le commentaire de C. Asdracha, ou encore ibid., no 91, p. 312-313 une inscription commémorant la restauration de l'église des Saints-Joachim-et-Anne, dans la région de Ganochôra, datée du 2 mai 1077. La date,

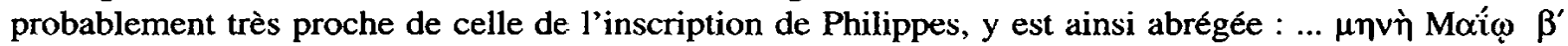

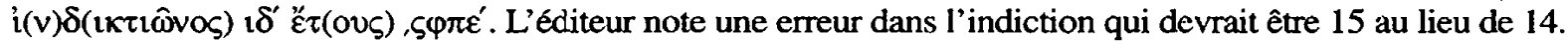

21. N. Zikos, op. cit., p. 282.

22. Pour Thessalonique, il s'agit de l'inscription de la porte maritime (voir infra, n. 26) ; à Philippes, voir l'inscription du kastron (citée supra, n. 4) ; pour Kavala, voir S. REINACH, La reconstruction des murs de Cavalla au Xe siècle, $B C H$ 61, 1882, p. 267-275. Dans son tout récent article de synthèse sur les fortifications byzantines de Macédoine, $\mathrm{K}$. Tsouris, qui reprend l'ensemble du dossier archéologique, cite les deux datations proposées pour l'inscription retrouvée à Drama sans trancher (K. TsOURIS, op. cit., p. 433).

23. VÉlÉNis \& TRIANIAPhyllidis, Drama, p. 104.

24. Ibid.

25. C'est aussi le calcul que fait N. Zikos (op. cit., p. 282). 
viendrait mieux à un ouvrage défensif qu'à un monument religieux. On peut ajouter que le linteau d'une porte du rempart maritime à Thessalonique ${ }^{26}$, daté de 904 , fournit un exemple comparable d'inscription gravée sur le linteau d'une porte fortifiée, avec un texte et un support très similaires.

Les éditeurs faisaient en conséquence du bloc retrouvé à Drama le linteau d'une porte des remparts de la ville médiévale. La deuxième partie de leur article est ainsi consacrée à l'examen des vestiges archéologiques de ces fortifications.

Cependant cette attribution ne repose sur aucun élément explicite issu du contexte archéologique de la découverte ni du texte lui-même. Le bloc a en effet été mis au jour fortuitement lors de travaux de voirie, à $400 \mathrm{~m}$ de distance du rempart oriental de la ville, dans une zone où la tradition locale situe un cimetière musulman 27 . Rien ne permettait d'en faire le linteau de la porte Est de la ville, mais aucun élément de l'inscription ne s'y opposait, et cette hypothèse restait la plus raisonnable.

Le texte lui-même ne contient en effet qu'un seul élément de quelque valeur topographique, la mention du métropolite de Philippes. Il ne serait certes pas étonnant de trouver trace à la fin du $11^{\mathrm{e}} \mathrm{s}$. d'une intervention de l'évêque de Philippes à Drama : comme l'a montré P. Lemerle, ce n'était alors qu'un bourg peu important, qui ne disposait pas de son propre évêque mais seulement d'un prôtopapas dépendant de l'évêché de Philippes 28 . Mais la présence d'une inscription monumentale de ce type (et les travaux qu'elle commémore) sur le rempart d'un simple bourg pourrait paraître pour le moins inhabituelle. Il semble bien d'autre part que la première fortification importante de Drama soit 1'œuvre de Boniface de Montferrat ${ }^{29}$ un siècle plus tard en 1206, même s'il existait déjà un rempart à la fin du $11^{\mathrm{e}}$ siècle.

Il n'est en revanche pas surprenant de trouver une telle inscription au-dessus d'une des portes de l'enceinte de Philippes, d'une tout autre échelle monumentale. La découverte à Philippes du fragment droit du linteau ne laisse pas de doute à ce sujet : c'est bien dans la métropole elle-même qu'il faut chercher l'emplacement originel du bloc retrouvé à Drama. Ce déplacement de quelque $22 \mathrm{~km}$ de la pierre ne doit pas étonner. Après son abandon au $15^{\mathrm{e}} \mathrm{s}$. - et probablement même avant les ruines de Philippes ont servi de carrière pour toute la région, comme en témoignent les premiers visiteurs ${ }^{30}$ du site et la dispersion d'inscriptions et

26. J.-M. SPIESER, Inventaire en vue d'un recueil des inscriptions historiques de Byzance. I. Les inscriptions de Thessalonique, TM 5, Paris 1973, no 12, p. 162. Le linteau de Thessalonique était d'une taille légèrement inférieure à celui de Philippes-Drama, avec $3,15 \mathrm{~m}$ de longueur pour $0,84 \mathrm{~m}$ de largeur et 0,27 $\mathrm{m}$ d'épaisseur. Il était aussi plus élaboré, avec une moulure (la seconde ligne est gravée sur un tore épais) et des ornements floraux à la fin de chaque ligne : le linteau de Philippes-Drama est en effet dépourvu de toute ornementation de ce type, ce qui constitue pour Vélénis et Triantaphyllidis un argument supplémentaire en faveur de son attribution à un ouvrage militaire.

27. Vélénis \& Triantaphyllidis, Drama, p. 100.

28. LeMERLE, Philippes, p. 259-260 et n. 1, p. 260. L'a. y note que l'essor de Drama date du $13^{\mathrm{e}} \mathrm{s}$. et que la ville n'est constituée en archevêché que sous les Paléologues.

29. Ibid. p. 180 ; VÉLÉNIS \& TRIANTAPHYLlidis, Drama, p. 112. L'attribution au rempart de Philippes de l'inscription trouvée à Drama retire à l'enceinte de cette dernière son unique attestation épigraphique, et le seul jalon chronologique antérieur au $13^{\mathrm{e}} \mathrm{s}$.

30. P. Belon au $16^{\mathrm{e}} \mathrm{s}$. assiste au démantèlement des gradins du théâtre revendus comme pierre de taille : cité par P. Collart, Philippes, ville de Macédoine depuis ses origines jusqu'à la fin de l'époque romaine, Paris 1937, p. 371. 
d'autres blocs sculptés dans de nombreux villages de la plaine de Drama. Ainsi, une tête colossale de Caracalla, trouvée à Drama et aujourd'hui au Louvre, provient très probablement du forum de Philippes ${ }^{31}$; deux chapiteaux à croix latine vus dans la même ville par B. Filov ${ }^{32}$ en 1913 appartenaient vraisemblablement à l'une des grandes basiliques de Philippes. Ce ne sont là que quelques exemples ${ }^{33}$. Tout comme les gradins du théâtre, le linteau de marbre dut constituer un morceau de choix pour les carriers à l'œuvre sur le site antique.

Si le fragment principal du linteau a donc beaucoup voyagé, l'autre partie n'a pas été abandonnée nécessairement non plus à son emplacement d'origine. Il ne fait pas de doute que la porte à laquelle il appartenait s'ouvrait dans un tronçon du rempart : l'analyse de G. Vélénis et $\mathrm{K}$. Triantaphyllidis garde sur ce point toute sa validité. De plus, des cinq chapelles mésobyzantines ${ }^{34}$ - on préférera ce terme à celui d'église, étant donnée la modestie des installations - connues à Philippes, aucune n'aurait pu accueillir un tel bloc. La plus proche d'entre elles, construite sur les ruines de la Basilique $D$, et distante seulement d'une centaine de mètres, au nord de l'emplacement actuel de la pierre, n'avait ainsi que $5 \mathrm{~m}$ à peine de largeur.

Les caractéristiques architecturales du réduit byzantin s'accommoderaient parfaitement d'une datation dans le dernier quart du $11^{\mathrm{e}} \mathrm{s}$. Il nous semble donc que la localisation actuelle du petit fragment du linteau reste significative : il devait appartenir à une porte de ce réduit. Une seule est connue, la Porte du Marais, dont l'origine remonte à la construction de l'enceinte macédonienne. Le linteau pourrait appartenir à une réfection de cette porte qui constituait probablement à l'ouest l'accès principal de la ville mésobyzantine en grande partie repliée sur le réduit fortifié. Mais il devait exister au moins une autre porte, vers l'intérieur de la ville cette fois, que la prospection topographique n'a malheureusement pas permis de localiser. Compte tenu de la topographie, notamment de la distribution des chapelles dans l'ancienne aire urbaine, on peut raisonnablement supposer que cette seconde porte s'ouvrait près du sommet du triangle qu'esquissc lc rcmpart byzantin, autrement dit à l'opposé de la Porte du Marais. Soit elle était alors située dans la courtine Est, et regardait alors vers la Basilique $B$, une hypothèse rendue intéressante par le grand redan que décrit la courtine à cet endroit et qui pourrait appartenir au dispositif d'une porte fortifiée ; soit elle était aménagée dans la courtine Nord, non loin de l'emplacement actuel de la pierre. En faveur de cette dernière hypothèse peut être mentionnée la présence, dans le même remblai que le linteau, d'un grand bloc soigneusement taillé et dont l'orientation est donnée

31. Ibid., p. 516.

32. B. FiLOV, Altchristliches aus Mazedonien, Studien sur Kunst des Ostens (Mélanges J. Strzygowski), Vienne 1913, p. 38 ; LEMERLE, Philippes, p. 411, n. 4.

33. On peut aussi rappeler que de manière plus discutable Y. Karayannopoulos a beaucoup usé de cet argument pour démontrer, à l'inverse, que les inscriptions protobulgares de la Basilique B n'ont pas été découvertes in situ : Y. KaRAYannopoulos, L'inscription protobulgare de Direkler, Athènes 1986, p. 47, n. 160. Cf. également les remarques de P. Lemerle : LemERLE, Philippes, p. 148.

34. Il s'agit, par ordre de découverte, de la chapelle du narthex de la Basilique $B$, de celles du sanctuaire de la Basilique D, du sanctuaire de la Basilique cimétériale, de l'Octogonc, ct cnfin de l'annexe Nord de la Basilique C. La chapelle de l'acropole semble quant à elle plutôt byzantine tardive, car contemporaine de l'érection du « donjon ». 
par un graffiti - une croix latine surmontée d'un signe non reconnu. Il pourrait s'agir d'un élément de chambranle de la porte monumentale.

La localisation de la porte grossièrement circonscrite, il reste à présent à élucider les circonstances des travaux du rempart que l'inscription commémore. Le verbe employé ( $\dot{\alpha} v \alpha \kappa \alpha \imath v i ́ \zeta \omega)$ n'est évidemment pas anodin : il s'agit d'une

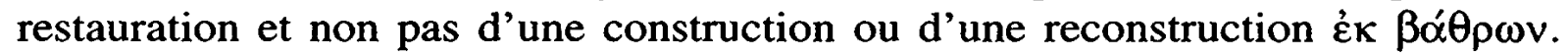
Cette précision confirme ce que détermine la diversité des appareils de maçonnerie observée dans la construction du réduit, et le redoublement de la courtine Sud-Est : le fortin a connu plusieurs phases, dont la première est antérieure à l'inscription, et remonte probablement au moins à la réfection des remparts de Philippes sous Nicéphore Phocas, un siècle plus tôt, vers 963-969. Contrairement en effet au premier commentaire ${ }^{35}$ qu'a donné $P$. Lemerle de l'inscription trouvée à Raktscha (Krinidès), il n'y a pas lieu de limiter aux fortifications de l'acropole la réfection probablement décidée par Nicéphore Phocas. Le terme de kastron peut aussi s'appliquer à toute petite ville fortifiée 36 . Il témoigne simplement du déclin de l'ancienne ville comme centre important de population au $10^{\mathrm{e}}$ siècle, et sa survie comme forteresse contrôlant un point de passage. Cette fonction même impliquait une restauration au moins partielle de l'enceinte de la ville basse, qui depuis la fondation de la ville barre le passage entre les marais et la colline, et dont les traces archéologiques sont encore visibles. Mais contrairement cette fois au second commentaire ${ }^{37}$ que P. Lemerle a fait des travaux sous Nicéphore Phocas, la création de remparts intermédiaires, sur les pentes de l'acropole, et dans la ville basse autour de la Porte du Marais, implique à notre avis que l'enceinte antique ne fut pas intégralement reconstruite ${ }^{38}$. Ce partitionnement de l'aire urbaine originelle ne peut en effet se comprendre que si le périmètre fortifié antique n'est plus entièrement défendu ni défendable.

La restauration dont il est question à la fin du règne de Michel Doukas fut vraisemblablement d'ampleur limitée : P. Lemerle ne voyait pour sa part pas trace d'une réfection des fortifications de Philippes entre Nicéphore Phocas et l'Empire latin ${ }^{39}$. En l'absence d'éléments de datation archéologiques que seule une série de sondages pourrait apporter, c'est dans l'inscription qu'il faut chercher quelque élément de réponse. Or contrairement à l'inscription du règne de Nicéphore Phocas, notre texte ne dit pas quel monument a bénéficié d'une rénovation partielle ou

35. LEMERLE, (n. 4), p. 104.

36. Ainsi Sparte est-elle qualifiée de « kastron de Lacédémone » dans une inscription de $1027: \mathrm{D}$. FeISSEL, A. PhILIPPIDIS-BRAAT, Inventaires en vue d'un recueil des inscriptions historiques de Byzance. III. Inscriptions du Péloponnèse, TM 9, Paris 1985, no 43, p. 300-302.

37. LeMERLe, Philippes, p. 148-150.

38. Le proteichisma, que Lemerle attribue aux ingénieurs de Nicéphore Phocas, est certainement bien antérieur et doit remonter à une réfection justinienne de l'enceinte, à une époque où se répand dans tous les Balkans ce dispositif défensif : cf. J. CROW, D. SMITH, The Hellenistic and Byzantine Defences of Tocra (Taucheira), Libyan Studies 29, 1998, p. 35-82. Voir aussi S. ProvosT, City wall and urban area in late antique Macedonia: a case study, Philippi, Recent Research in Late Antique Urbanism, JRA Supplement 43, 2001, p. 123-135. K. TsouRIS (op. cit., p. 425-426 et n. 96) conteste lui aussi la chronologie propusée par Lemerle pour l'enceinte basse, mais lui assigne une datation antérieure à Justinien ( $3^{\mathrm{e}}-5^{\mathrm{e}}$ s. ap. J.-C.).

39. LeMERLe, Philippes, p. 150. 
entière. Sans être exceptionnel, le fait est tout de même inhabituel dans une inscription assez développée 40 : les commanditaires de ces prestigieux et coûteux travaux précisent généralement l'objet de leur intervention s'il est limité à une partie seulement du monument (porte, tour) ou soulignent au contraire l'ampleur d'un programme plus général ${ }^{41}$. En l'absence de sujet, l'inscription désigne au lecteur son support, dans un flou qui permet une interprétation flatteuse de la réalité des travaux. Dans le cas du linteau de Philippes, cette imprécision renforce l'impression d'une restauration limitée, soit au réduit byzantin de la Porte du Marais, soit même simplement à la porte fortifiée en question. Il ne s'agit pas en tout cas du kastron tout entier. Deux exemples d'inscription commémorant la construction ou restauration d'une porte de rempart ont déjà été cités : à Apros, la porte est mentionnée ${ }^{42}$, mais pas à Thessalonique ${ }^{43}$, dont l'inscription est probablement celle qui se rapproche le plus de celle de Philippes.

Plus encore que l'imprécision du sujet, c'est le responsable des travaux mentionné dans l'inscription qui surprend : le métropolite Basile Kartzimopôlos. Ce personnage était inconnu jusqu'à la découverte de l'inscription de Drama. Il $s$ 'insère dans les fastes de la métropole de Philippes entre deux évêques mal connus et mentionnés par une seule source douteuse ${ }^{44}$, Damien en 1072 et Nicétas en 1098. G. Vélénis et K. Triantaphyllidis relient l'étymologie de son nom au terme $\kappa \alpha \rho \tau \zeta \mu \alpha \hat{\alpha} \varsigma$ (eunuque) et l'utilisaient comme élément de datation de l'inscription ${ }^{45}$.

Le texte le désigne comme le responsable direct des travaux, ce qui est inattendu dans le cas d'un métropolite pour la rénovation d'un ouvrage fortifié. Les inscriptions mésobyzantines ne manquent pas qui commémorent les réalisations d'évêques, mais il s'agit dans l'immense majorité des cas, de monuments ecclésiastiques. Dans l'Antiquité tardive, lorsque l'évêque en vient à se substituer en grande partie aux autorités civiles locales, une telle responsabilité ne surprendrait

40. Par opposition avec les simples acclamations ou «signatures » (souvent un simple monogramme) qu'on trouve en nombre sur les ouvrages de défense : voir par exemple, la série d'inscriptions du kastron de Didymoteichon en Thrace, dans C. AsDraCHA - Ch. BAKIRTZIS, Inscriptions byzantines

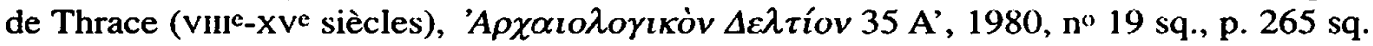

41. Voir par exemple, pour les inscriptions déjà mentionnées, la porte d'Apros dans le premier cas (infra n. 42) et le kastron fondé par Constantin Diogène dans le second (supra n. 16).

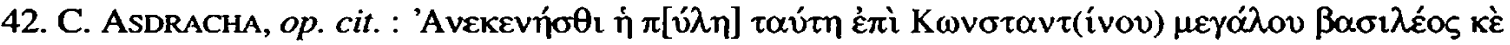

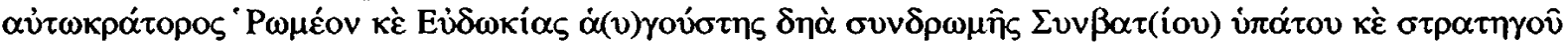

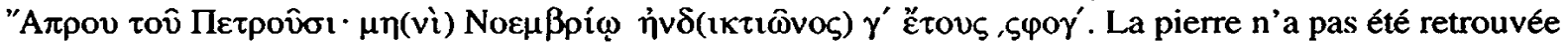
à Apros mais dans un village des environs. L'éditeur n'en considère pas moins que le texte se réfère à une porte de la muraille d'Apros, notamment en raison de l'implication du stratège de la ville et de la mention des empereurs régnants.

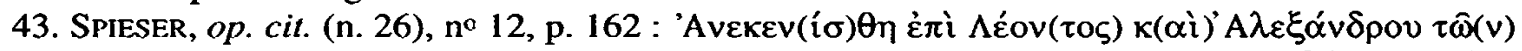

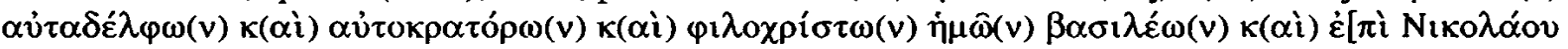

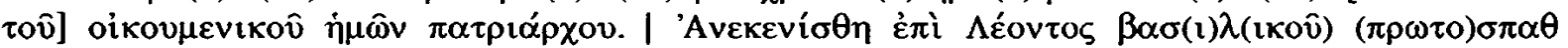

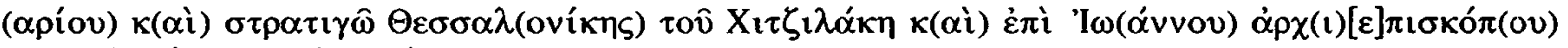

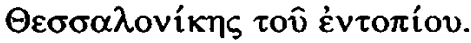

44. Il s'agit d'une courte monographie sur Philippes, écrite en 1899 par S. Mertzidès, comportant une liste épiscopale, dépourvue de référence précise pour les deux évêques en question : il est cité par P. Lemerle, Philippes, p. 272.

45. Vélénis \& TRIANTAPHYLlidis, op. cit., p. 101. Leur démonstration nous paraît plus convaincante que l'hypothèse de N. ZıKos (op. cit., p. 282) qui, pour sa part, donne une restitution légèrement différente du nom abrégé, Kartzimopolitou, et en fait un ethnique. 
pas : nombre d'inscriptions sur des édifices publics civils et militaires en témoignent ${ }^{46}$. Aux $9^{e}-12^{e}$ s. en revanche, le cas paraît exceptionnel. Il peut arriver comme à Thessalonique dans le cas de la porte maritime, que le métropolite soit cité aux côtés du fonctionnaire impérial, mais le responsable des travaux est bien ce dernier, à savoir ici le stratège de Thessalonique Léon Chitzilaki47.

L'épigraphie et les sources littéraires tendent donc à montrer qu'au $11^{\mathrm{e}} \mathrm{s}$. une telle responsabilité incombe au stratège ou au duc du thème correspondant : outre le kastron déjà mentionné fondé par le katépan ${ }^{48}$ de Thessalonique Constantin Diogène en 101549, on peut encore citer l'exemple de Michel, duc de Crète, restaurant le kastron de Hiéron 50 . Pour le rempart ou la porte fortifiée de Philippes à la fin du $11^{\mathrm{e}} \mathrm{s}$., on attendrait donc la mention du duc du thème de Thessalonique, du Strymon et du Boléron, ou de l'un de ses subordonnés, puisque ces thèmes ont fusionné depuis le milieu du siècle 51 . Peut-on expliquer son absence et l'implication du métropolite par des circonstances politiques ou militaires particulières ?

La liste des ducs de Thessalonique connus au $11^{\mathrm{e}} \mathrm{s}$. est relativement bien fournie, surtout dans la première moitié du siècle ${ }^{52}$. Mais les lacunes couvrent presque entièrement le règne de Michel VII, qui nous intéresse ici : l'avant-dernier duc attesté n'est autre que Nicéphore Botaniatès, en 1062, celui-là même qui renversa Michel Doukas en 1078. On ne connaît donc pas de duc de Thessalonique pour 1076-1078, et ce silence des sources non seulement épigraphiques ou sigillographiques mais aussi littéraires est intéressant. Le thème est en effet au centre d'une région où se développent deux importantes révoltes militaires 53 à partir de l'automne 1077 : c'est en effet en octobre 1077 que Michel VII (ou plutôt son ministre Nicéphoritzès) remplace le duc de Dyrrachium, Nicéphore Bryennios, par Nicéphore Basilakios. Bryennios rentre alors chez lui, à Andrinople, d'où il prend de facto le contrôle du thème de Macédoine et de Thrace ${ }^{54}$, et monte une

46. Sur le sujet, voir l'étude de D. FEISSEL, L'évêque, titres et fonctions d'après les inscriptions grecques jusqu'au viIe siècle, Actes du XI Congrès International d'Archéologie Chrétienne, Lyon, Vienne, Grenoble, Genève et Aoste (septembre 1986), Rome 1989, p. 801-828.

47. Voir supra n. 43.

48. L'équivalence entre les titres de duc et katépan est à présent généralement admise : J.-Cl. Cheynet, Du stratège de thème au duc : chronologie de l'évolution au cours du XIe siècle, TM 9, 1985, p. 181. Voir aussi H. AhrWeILeR-Glykatzi, op. cit., p. 64-65.

49. Voir supra, n. 16.

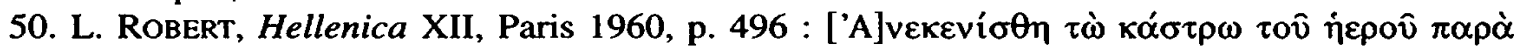

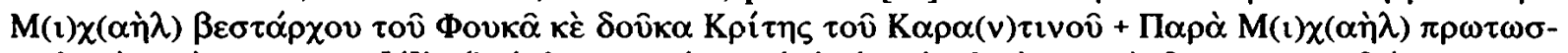

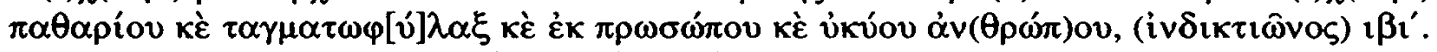

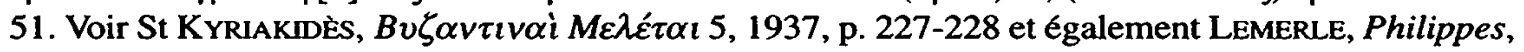
p. $159-168$.

52. Elle a d'abord été établie par St Kyriakidès, puis revue par P. LEMERLE, Philippes, p. 152-159. Consulter également, pour la première moitié du siècle, J.-Cl. CHEYNET, Pouvoir et contestations à Byzance (963-1210), Paris 1990, p. 308.

53. Voir J.-Cl. CHEYNET, op. cit., p. 351-353 sur le caractère régionaliste de ces deux rébellions et l'analyse des différents clans et solidarités des protagonistes.

54. Il y avait alors à Andrinople un katépan, parent par alliance de Bryennios auquel il se rallia : ATtaleiatès, éd. Bonn, p. 242. Le thème de Macédoine et de Thrace correspond géographiquement à la Thrace et ne correspond en rien aux provinces antiques de Macédoine, qui sont incluses dans le thème de Thessalonique. La ville la plus occidentale passée aux mains de Bryennios semble avoir été Traianoupolis. Sur ce point, voir C. AsDRACHA, op. cit. (n. 9), p. 314-315. 
révolte contre l'empereur qui conduira son frère Jean à tenter une attaque de Constantinople au mois de novembre suivant 55 . Peu de temps après, c'est au tour de Basilakios d'entrer en rébellion contre l'empire. Il en découle qu'à la fin de l'année 1077 et au début de 1078, le thème de Dyrrachium et celui de Macédoine et de Thrace sont contrôlés par des usurpateurs. Le thème de Thessalonique est alors isolé et coupé de la capitale, sans qu'on sache si ses troupes et son administration restèrent fidèles ou non à l'empereur. Il est possible qu'une partie des tagmata du thème ait combattu aux côtés de Nicéphore Bryennios dans la bataille finale contre Alexis Comnène, probablement en mai 1078, si c'est bien ainsi qu'il faut interpréter la mention chez l'historien Nicéphore Bryennios, reprise par Anne Comnène, d'un corps de troupes macédoniennes ${ }^{56}$. L'influence de Bryennios ne dépassa pas la Thrace occidentale, où il s'employa à faire restaurer un certain nombre de fortifications, comme en témoigne l'épigraphie 57.

De son côté en revanche, Basilakios s'empare apparemment sans coup férir de Thessalonique, au printemps 1078 , et nulle mention n'est faite à cette occasion du duc ou de quelque autre représentant du pouvoir impérial dans les sources littéraires pourtant nombreuses de ces événements. Basilakios étendit même peutêtre son influence jusqu' aux limites occidentales du thème : lorsque Alexis Comnène, après avoir vaincu Bryennios pour le compte de Nicéphore Botaniatès, est envoyé combattre Basilakios, il doit en chemin se défaire d'une garnison, envoyée en avant-poste par ce dernier à Périthéôrion ${ }^{58}$. On notera avec intérêt que cette ville située à l'est de Philippes, sur une rive du Nestos, en est encore à l'époque un évêché suffragant ${ }^{59}$. C'est aussi au milieu du $11^{\mathrm{e}}$ siècle la ville principale d'un $\beta \alpha^{\prime} \delta \delta$, subdivision du thème du Boléron ${ }^{60}$. Il est donc possible que la région de Philippes soit passée brièvement sous contrôle de Basilakios, mais 1'hypothèse est difficile à confirmer : cette brève note d'Attaleiatès est en effet le seul témoignage d'une activité militaire de Basilakios à l'est de Thessalonique.

Vaincu en juillet 1078 par Alexis Comnène ${ }^{61}$, Basilakios est ramené captif vers la capitale quand il rencontre les envoyés impériaux porteurs d'un prostagma ordonnant de crever les yeux du rebelle62. Attaleiatès précise que cette rencontre

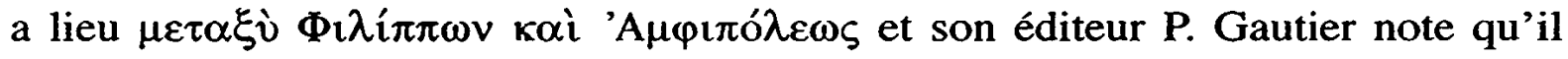

55. D. Polemis, Notes on Eleventh-century chronology (1059-1081), BZ 58, 1965, p. 68 ; ATTALEIATĖs, ed. Bonn, p. 247-250 ; BRYENNIOS, p. 114.

56. BRYEnNIOS, p. 136. Voir C. ASDRACHA (op. cit. (n. 9), p. 314-315) sur l'ambiguïté de l'expression.

57. C. AsDRACHA, op. cit. (n. 9), no 92, p. 313-315, pour le cas d'Andrinople.

58. Atraleiatès, éd. Bonn, p. 299, suivant la lecture que P. Gautier (cf. Nicephori Bryennii historiarum libri quattuor, Introduction, texte, traduction et notes par P. GAUTIER, CFHB, series Bruxellensis IX, Bruxelles 1975, p. 284, n. 5) fait de ce passage : (il s'agit d'Alexis Comnène) òs

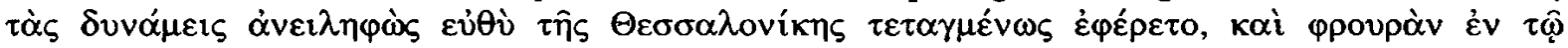

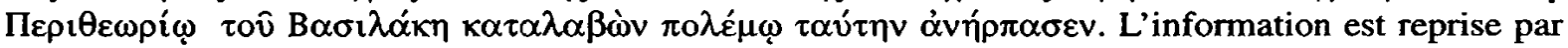
SCYLITZĖs CONTINUATUS, p. 183.

59. Lemerle, Philippes, p. 261. Contra voir P. Soustal, Tabula Imperii Byzantini 6, Thrakien, Vienne 1991 , p. 394.

60. Ibid., p. 163.

61. La date, comme l'ensemble de la chronologie de la révolte de Basilakios, n'est pas certaine : cf. Cheynet, op. cit. (n. 52), p. 85 et 86, n. 5 .

62. Attalelatès, p. 156. Cf. la note de Gautier, Bryennios (n. 58), p. 296, n. 4. 
existe encore dans la région de Kavala un village appelé Basilakion ${ }^{63}$, dont le nom pourrait ainsi rappeler l'événement. Cette mention de Philippes, la seule dans toutes les sources historiques consacrées aux événements de 1077-1078, a pour mérite de souligner que la ville fortifiée constitue toujours une étape importante sur la route de Constantinople à Thessalonique. Et cette route fut presque certainement empruntée par tous les protagonistes de cette double révolte. Ainsi la description par Bryennios ${ }^{64}$ de l'itinéraire de campagne d'Alexis Comnène au printemps 1078 le montre traversant la Macédoine et le Boléron jusqu'au Strymon avant de remonter vers le Nord.

Bien que la chronologie de ces événements reste parfois imprécise, les travaux de fortification à Philippes n'ont probablement pas de lien direct avec eux, puisque l'inscription, datée de 6585 , est antérieure au $1^{\text {er }}$ septembre 1077 . S'ils n'étaient pas nécessairement achevés au moment de la rébellion, ces travaux n'en avaient pas moins été décidés avant son déclenchement. Il n'est donc pas possible de faire des travaux engagés par le métropolite Kartzimopôlos à Philippes cette année-là une réaction loyaliste locale face aux rébellions provinciales voisines 65 . En revanche, il n'est pas interdit d'y voir tout de même le signe exceptionnel d'une certaine passivité ou incapacité de l'administration provinciale qui conduisit le hiérarque à prendre au moins partiellement en charge la défense de sa métropole. C'est dans ce contexte qu'il était intéressant de rappeler en détail les événements. L'absence curieuse dans les sources de ces deux rebellions provinciales d'une mention des responsables administratifs du thème de Thessalonique rencontre d'une certaine manière une confirmation dans l'initiative locale que représentent les travaux commémorés par l'inscription de Philippes.

Les invasions de la seconde moitié du $11^{\mathrm{e}} \mathrm{s}$, notamment des Petchénègues et des Coumans, ont contraint l'empire à restaurer nombre de fortifications dans les Balkans, comme en témoignent diverses sources littéraires et épigraphiques. Faute de disposer des finances nécessaires, Michel VII autorisa même la donation pour une durée limitée de kastra dont il ne pouvait probablement pas assurer l'entretien ou la restauration 66 . Tel n'est certes pas le cas de Philippes, mais la mesure montre que l'empereur devait encourager toutes les initiatives locales de rénovation, et l'intervention du métropolite en 1077 s'inscrit peut-être dans cette perspective. Il est vrai que les sources ne mentionnent pas de menace directe contre la ville dans les années 1070 , mais les régions voisines au nord sont troublées par les Petchénègues, particulièrement entre 1071 et 107467 . L'alerte a pu paraître suffisamment

63. Ibid., p. 296, n. 5.

64. BRYENNIOS, p. 148.

65. J.-Cl. Cheynet note toutefois qu'en province au $11^{\mathrm{e}} \mathrm{s}$. les evêques se gardaient bien en général de suivre les rebelles, et avaient plutôt une attitude loyaliste ou du moins modératrice, qui parfois put même leur coûter la vie : CHEYNET, op. cit. (n. 52), p. 317.

66. 1 bid., p. 307 et N. OIKONOMIDES, The donation of castles in the last quarter of the $\mathrm{xt}^{\text {th }}$ century, Polychronion. Heidelberg 1996, p. 413-417.

67. P. DiACONU, Les Petchénègues au Bas-Danube, Bucarest 1970, p. 100-109. Voir Egalement J. FINE, The Early Medieval Balkans, A Critical Survey from the Sixth to the Late Twelfth Century, Ann Arbor 1983, p. 211. 
chaude pour entraîner des travaux de fortifications dans certaines villes de Macédoine, parmi lesquelles Philippes. Les Petchénègues continuent en effet de faire peser une menace et ils jouent un rôle mineur dans les événements de 1077-1078, puisqu'ils profitent des tentatives d'usurpation pour attaquer le thème de Thrace et de Macédoine : ils mettent même le siège devant Andrinople et contraignent Nicéphore Bryennios à la négociation 68 .

L'inscription de Philippes s'inscrirait donc dans le cadre d'une restauration générale des villes fortifiées des provinces balkaniques dans la deuxième moitié du $11^{\mathrm{e}}$ siècle entraînée par une insécurité grandissante apportée par les invasions des Petchénègues et des Coumans. Outre le témoignage capital qu'elle apporte de l'existence du site à cette époque tardive, son originalité réside dans le commanditaire des travaux de fortification, le métropolite et non le duc du thème. Il est possible d'y voir le résultat des circonstances politiques troublées de 1077, mais cette hypothèse reste difficile à prouver.

Samuel Provost

École française d'Athènes

68. AtTaleiatès, p. 262 ; P. Diaconu, op. cit., p. 109-110, estime que les Petchénègues agissaient en cette occasion comme les alliés de l'empereur Michel VII Doukas. 